\title{
Non-invasive, Spatiotemporal Characterization of Muscle Activation Patterns from Vagus Nerve Stimulation in Human Subjects Maria Feucht ${ }^{1}$, Matthew Ward ${ }^{1,2}$ \\ ${ }^{1}$ Indiana University School of Medicine, ${ }^{2}$ Weldon School of Biomedical Engineering, Purdue University
}

Background and Hypothesis: Vagus nerve stimulation (VNS) is used for treatment of epilepsy in over 100,000 patients worldwide and is a potential treatment for many inflammatory disorders. VNS-evoked compound nerve action potentials (CNAPs) may have applications as a biomarker of VNS treatment efficacy, but transcutaneous measurement of this activity is obscured by muscle artifacts. A more precise understanding of muscle activation patterns could improve recording and analysis protocols for isolating the vagus nerve (VN) CNAP. We hypothesize that analyzing multi-electrode array (MEA) surface recordings overlying the $\mathrm{VN}$ will allow us to characterize this muscle artifact.

Project Methods: Five patients undergoing VNS therapy for epilepsy were enrolled in a clinical study at Indiana University School of Medicine (IRB \#2006075899). A custom-made MEA with a grid of 32 soft foam electrodes was placed on the skin overlying the $\mathrm{VN}$ on each side of the neck. Surface potentials were recorded for approximately 20 minutes at the patient's established device settings.

Results: VNS-evoked potentials were visualized in all five patients. Two probable muscle artifacts were identified, defined as non-propagating features in the mean response to $n>600$ pulses of VNS. The first had an onset latency of $\sim 1-3 \mathrm{~ms}$, and the second had a latency of $\sim 7-$ $10 \mathrm{~ms}$. Both artifacts appeared primarily in the electrodes overlying the laryngeal muscles.

Conclusion and Potential Impact: The short latency of the first muscle artifact suggests stimulus signal leakage activating the superior laryngeal nerve. The second artifact with longer latency is likely caused by the recurrent laryngeal nerve. Previous analyses may have mischaracterized the first muscle artifact as nerve activity. The use of MEA-based recordings clarifies our understanding of the VN's response to VNS, which may lead to better treatment efficacy and the eventual development of personalized VNS therapies for epilepsy and a range of inflammatory disorders. 